\title{
Vascular endothelial growth factor in articular cartilage of healthy and osteoarthritic human knee joints
} D Pfander, D Körtje, R Zimmermann, G Weseloh, T Kirsch, M Gesslein, T Cramer,
B Swoboda
Medical Centre,

Hershey, PA, USA

$\mathrm{T}$ Kirsch

Department of Gastroenterology, Virchow-Klinikum, Humboldt-University of Berlin, Berlin, Germany

T Cramer

Correspondence to: Dr D Pfander, Division of Orthopaedic Rheumatology, Department of Orthopaedic Surgery im Waldkrankenhaus St Marien, University of Erlangen-Nuernberg, Rathsbergerstr 57, D-91054 Erlangen, Germany

Dpfander@t-online.de

Accepted 12 April 2001

Osteoarthritis (OA) is a multifactorial disease of diarthrodial joints, resulting in a loss of articular cartilage, a remodelling of subchondral bone, and inflammatory reactions of the synovialis. ${ }^{1}$ The key event in OA cartilage is the failure of chondrocytes to compensate for the enzymatically and mechanically induced matrix degradation. ${ }^{23}$

Vascular endothelial growth factor (VEGF) was found to be present in the hypertrophic zone of the embryonic mouse growth plate cartilage. Evidence was provided that VEGF produced by hypertrophic chondrocytes stimulates the angiogenesis in growth plate cartilage. ${ }^{4}$ Four different VEGF isoforms, consisting of $121,165,189$, and 206 amino acids, respectively, result from alternative splicing of a single gene product. $^{5}$ In addition, four VEGF

\begin{abstract}
Division of
Orthopaedic

Rheumatology,

Department of

Orthopaedic Surgery,

University of

Erlangen-Nuernberg,

Erlangen, Germany

D Pfander

D Körtje

R Zimmermann

G Weseloh

M Gesslein

B Swoboda

Department of Orthopaedics, Penn

State College of

Medicine, Hershey

Abstract

Objective-To determine the levels of vascular endothelial growth factor (VEGF) mRNA and protein expression in normal and osteoarthritic (OA) human articular cartilage, and whether VEGF expression alters during the progression of $O A$.

Methods-Sections from normal and OA human knee cartilage were immunotained with a polyclonal antibody recognising VEGF. In addition, total RNA was isolated from normal and osteoarthritic human knee cartilage and analysed by reverse transcriptase-polymerase chain reaction (RT-PCR) for VEGF MRNA expression.

Results-VEGF was found to be present in normal and OA human knee cartilage in all cartilage layers. A significant increase of VEGF immunopositive chondrocytes to up to $\sim 82 \%$ was detected in severe OA cartilage compared with normal articular cartilage ( $56 \%$ of immunopositive chondrocytes). RT-PCR analysis showed the expression of VEGF also on the mRNA level.

Conclusions-VEGF is expressed by articular chondrocytes in normal and $O A$ human knee cartilage. The percentage of VEGF immunopositive chondrocytes significantly increases in late stages of the disease. The VEGF transcript levels encoding all four isoforms shows a big variability in samples from different donors, suggesting a distinct regulation of the expression of the four VEGF isoforms in normal and $O A$ cartilage.

(Ann Rheum Dis 2001;60:1070-1073)
\end{abstract}

receptors have been characterised. ${ }^{6}$ Previous studies have shown an increased production of VEGF under hypoxic conditions and in response to mechanical forces. ${ }^{78}$ Interestingly, altered oxygen pressures and mechanical forces have been demonstrated in articular cartilage under pathological conditions, such as OA. ${ }^{910}$ Thus, possibly, an increased expression of VEGF occurs in OA cartilage, leading to the observed blood capillary invasion from subchondral bone into articular cartilage. To test this hypothesis we considered the following questions: (a) Do articular chondrocytes in vivo express VEGF? (b) Does VEGF expression correlate with the severity of OA?

\section{Materials and methods}

SAMPLE ACQUISITION, TISSUE PREPARATION, AND HISTOLOGICAL/HISTOCHEMICAL GRADING

Twenty seven OA human cartilage samples from 26 different donors were obtained from patients undergoing total knee replacement. The clinical data were carefully reviewed to exclude any forms of secondary OA and inflammatory joint diseases. Additionally, six healthy samples without any signs of OA were obtained from four donors within 12-24 hours after death and from one patient who underwent an amputation. Specimens were fixed, decalcified, and embedded in paraffin as described. ${ }^{11}$ After sectioning, specimens were stained with safranin $\mathrm{O}$, scored according to Mankin, and divided into normal, mild OA, moderate OA, and severe OA as described. ${ }^{11}{ }^{12}$

\section{IMMUNOHISTOCHEMISTRY}

After deparaffinisation, non-specific binding sites were blocked by incubation with 5\% bovine serum albumin in phosphate buffered saline $\mathrm{pH} 7.4$ for 30 minutes. Then sections were incubated overnight at $4^{\circ} \mathrm{C}$ with a polyclonal antibody against VEGF (IgG) in a dilution of 1:100 (Santa Cruz Biotechnology Inc, USA). Control cartilage sections were incubated with non-immune rabbit IgGs (Santa Cruz Biotechnology Inc, USA). After incubation with primary antibodies, sections were treated with biotinylated donkey antirabbit antibodies (Dianova, Hamburg, Germany), followed by streptavidin labelled with alkaline phosphatase (Dako, Hamburg, Germany) and staining with Fast Red (Sigma, Munich, Germany). Sections were viewed under a Leica microscope. The percentage of immunopositive cells was determined by counting 100 chondrocytes twice throughout 
the entire depth of cartilage under a high power field. Statistical analyses were carried out on all data points for immunopositive cells in normal articular cartilage by a non-parametric MannWhitney U test. Data are given as means (SD) of different donors. p Values less than 0.05 were considered significant.

RNA PREPARATION

Seventeen samples of OA cartilage were obtained from 12 patients who were undergoing a total knee replacement. Additionally, two samples with no signs of joint disease were obtained from necropsies. The tissue was frozen and shredded in liquid nitrogen. Total
RNA was isolated from cartilage using the TRIzol reagent (BRLGibco, Eggenstein, Germany) and purified with the Nucleo-SpinRNA-II-Kit (Clontech Laboratories, Palo Alto, USA), as recommended by the manufacture. Total RNA yields were calculated by measurement of the absorbance at $260 \mathrm{~nm}$ (UltrospecIII, LKB).

REVERSE TRANSCRIPTION (RT) AND POLYMERASE CHAIN REACTION (PCR)

Sequences of VEGF primers were designed as described by Iizuka et al. ${ }^{13}$ The primers used had the following sequences: 5'-CTGCTGT CTTGGGTGCATTG-3' and 5'-TTCACAT
A
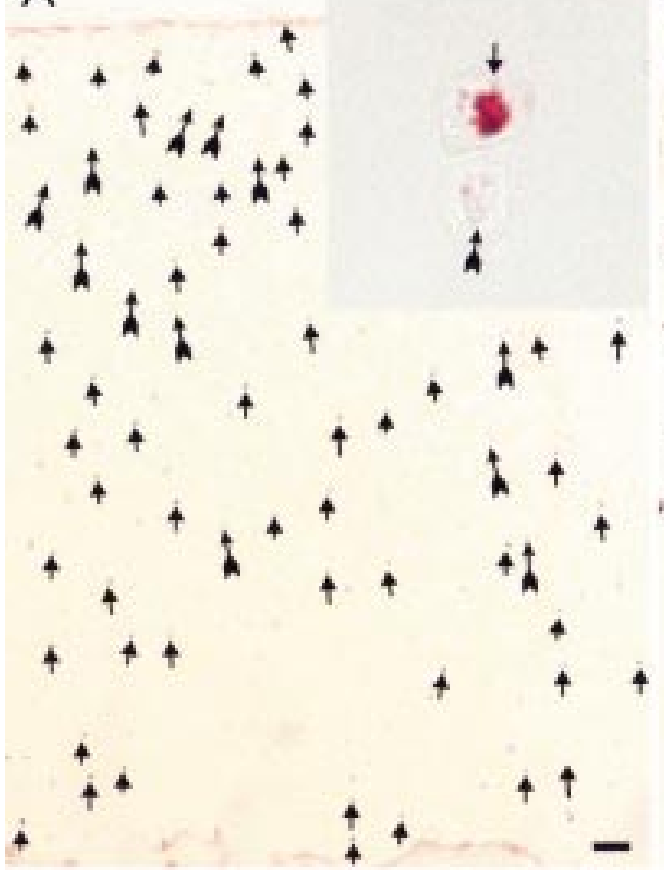

C
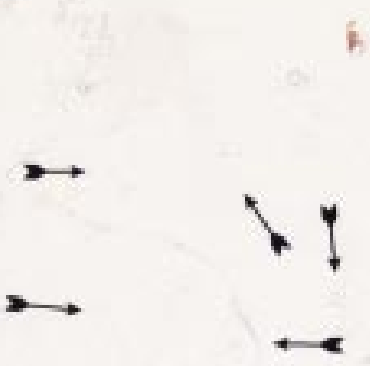

5

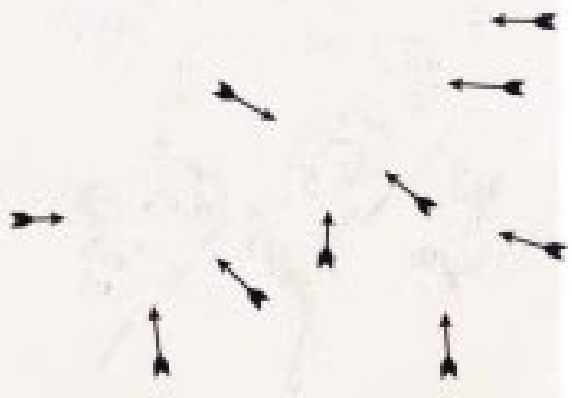

Figure 1 (A) Normal articular cartilage immunostained with antibodies against VEGF, showing positive (arrowheads) and negative chondrocytes (arrows) in all cartilage layers; Mankin score 1. VEGF positive and negative chondrocytes from the deep zone are shown in close up (inset). (B) Severe OA cartilage with a strong immunostaining of the chondrocyte clusters next to the surface (arrowheads) and also VEGF positive chondrocytes throughout the remaining deep zone (arrows); Mankin score 11. Positive chondrocytes within a cluster are displayed in close up (inset). (C) A slide adjacent to (B) incubated with non-immune rabbit serum as negative control. Arrows indicate negative chondrocytes. Bar represents: (A) $200 \mu \mathrm{m}$; (B) $100 \mu \mathrm{m}$; and (C) $60 \mu \mathrm{m}$. 
TTGTTGTGCTGTAG-3'. The resulting PCR product was $378 \mathrm{bp}$. RNA $160 \mathrm{ng}$ was converted to complementary DNA (cDNA) by incubation with specific reverse primers for VEGF. The RT reaction was performed using the first strand cDNA synthesis kit (Roche Diagnostics GmbH, Mannheim, Germany) in a $20 \mu \mathrm{l}$ reaction mixture $(5 \mathrm{mM} \mathrm{MgCl}, 1 \mathrm{mM}$ dNTP mix, RT buffer, inhibitor, and $20 \mathrm{U}$ AMV reverse transcriptase ) for 10 minutes at $25^{\circ} \mathrm{C}$, followed by incubation at $42^{\circ} \mathrm{C}$ for 60 minutes, and enzyme denaturation at $95^{\circ} \mathrm{C}$ for five minutes. cDNA $2 \mu \mathrm{l}$ was amplified by PCR through 29 or 35 cycles (denaturation at $95^{\circ} \mathrm{C}$ for 30 seconds, annealing at $60^{\circ} \mathrm{C}$ for 30 seconds, and elongation at $72^{\circ} \mathrm{C}$ for 45 seconds) using taq-DNA-polymerase (Roche
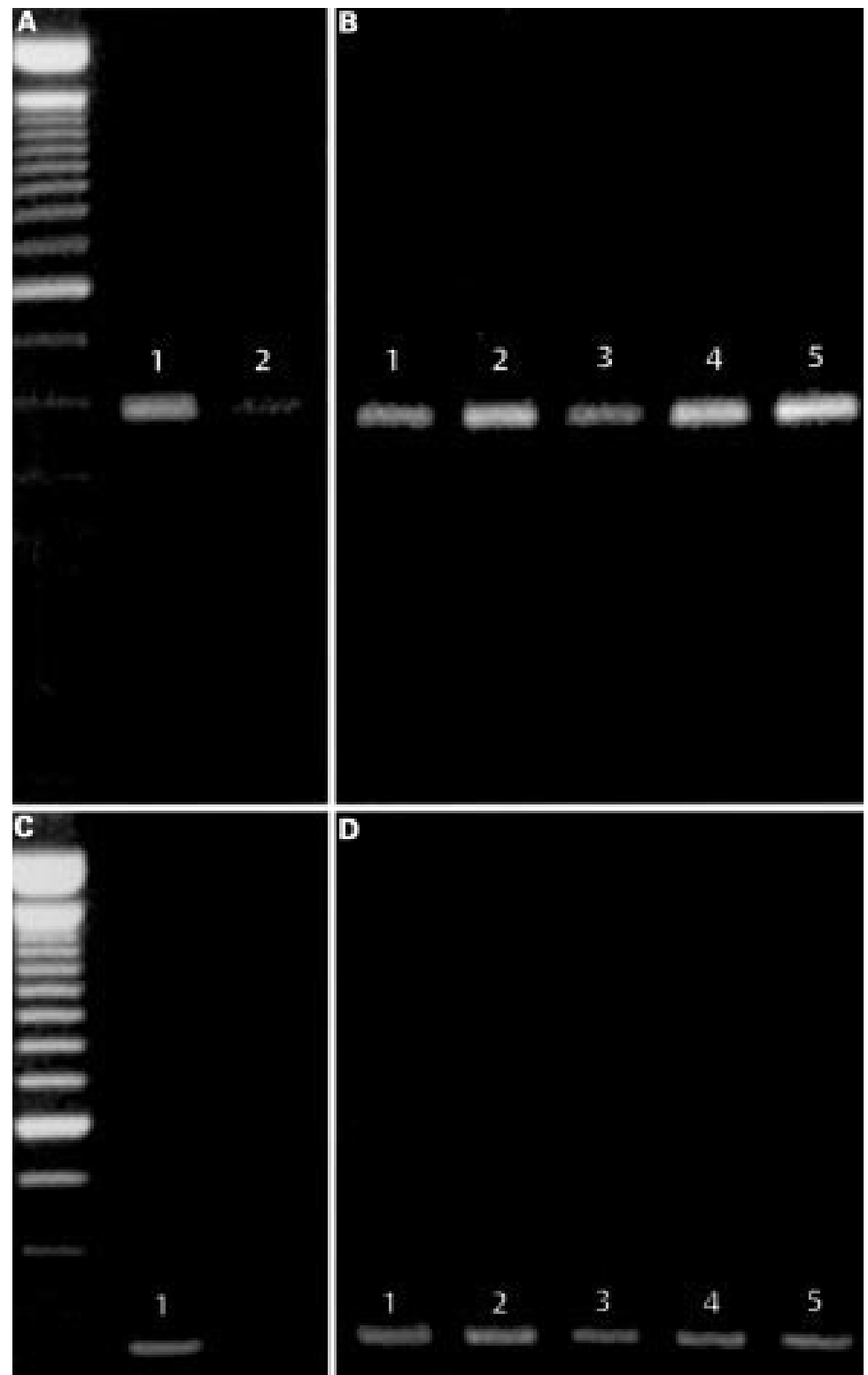

Figure 2 Reverse transcriptase-polymerase chain reaction (RT-PCR) analyses of VEGF $m R N A$ levels compared with corresponding GAPDH $m R N A$ levels in human normal and $O A$ samples. Resulting PCR products were analysed on a $1.5 \%$ agarose gel. (A) VEGF in normal cartilage. Lanes 1 and 2 represent the PCR product ( $378 \mathrm{bp}$ ) of the same sample with 35 or 29 cycles, respectively. (B) PCR products of different OA cartilage samples after 35 cycles; lane 1 represents a grade I lesion, lanes 2 and 3 show grade II lesions, lanes 5 and 6 demonstrate grade III lesions. (C and D) represent the corresponding GAPDH PCR products (294 bp) of the same donors as shown above.
Table 1 Summarised results of vascular endothelial growth factor (VEGF) immunostaining

\begin{tabular}{ll}
\hline & $\begin{array}{l}\text { Percentage of VEGF } \\
\text { positive chondrocytes }\end{array}$ \\
\hline Normal cartilage samples $(\mathrm{n}=6)$ & $56(13.0)$ \\
Mild OA samples $(\mathrm{n}=8)$ & $51.4(11.2)$ \\
Moderate OA samples $(\mathrm{n}=9)$ & $53.2(12.8)$ \\
Severe OA samples $(\mathrm{n}=10)$ & $82.2^{\star}(6.9)$
\end{tabular}

Percentages of VEGF positive chondrocytes throughout the entire depth of the specimens. In each specimen 100 chondrocytes were counted twice using a high power magnification field. Percentage of VEGF immunostained chondrocytes are given as means (SD).

${ }^{\star} \mathrm{p}<0.05$ (versus percentage of positive chondrocytes in normal cartilage).

Diagnostics GmbH, Mannheim, Germany) and the specific upstream and downstream primers $(0.4 \mu \mathrm{M})$ in a $25 \mu \mathrm{l}$ reaction mixture containing $2 \mathrm{mM} \mathrm{MgCl}_{2}$ and $0.2 \mathrm{mM}$ Nucleotide-Mix (Boehringer, Mannheim, Germany). The PCR was terminated by an elongation step for 10 minutes at $72^{\circ} \mathrm{C}$. To estimate the amount of VEGF mRNA, GAPDH mRNA levels were determined. Therefore, RNA was transcribed into single complementary DNA using AMV reverse transcriptase (Boehringer, Mannheim, Germany) and oligo-p $(\mathrm{dT})_{15}$ primer, according to the manufacturers protocol. GAPDH primers used in the amplification reactions had the following sequences 5'-CGAGATCCCTCCAA AA TCAA-3' and 5'-TGTGGTCATGAGT CCCA-3', resulting in a product of $294 \mathrm{bp}$. cDNA was amplified through 35 cycles. PCR products were separated on $1.5 \%$ agarose gels and visualised by ethidium bromide staining.

\section{Results}

Expression of VEGF on the protein level in normal and OA human articular cartilage samples was determined by immunohistochemistry with antibodies recognising predominantly the 165 amino acid long VEGF isoform. Chondrocytes immunopositive for VEGF were detected in all layers of normal and OA cartilage (fig 1A). There was a significant increase in the number of VEGF positive chondrocytes in cartilage samples of severe OA. Especially, enlarged clustered chondrocytes next to the cartilage surface showed strong immunostaining for VEGF (fig 1B). Slides incubated with non-immune rabbit IgGs showed no staining (fig 1C). In mild and moderate OA cartilage a slight, but non-significant reduction of VEGF stained chondrocytes was detected. This decrease was mainly due to a smaller number of VEGF positive chondrocytes in the deep zone. To determine whether VEGF immunopositive chondrocytes express VEGF also on the mRNA level, we isolated total RNA from normal and OA human cartilage samples and analysed VEGF expression by relative quantitative RT-PCR analysis. The primers used for PCR analysis detected all four isoforms of VEGF. The levels of VEGF mRNA expression in healthy and OA cartilage varied significantly between samples from different donors without a clear correlation with OA progression (fig 2). Table 1 summarises the results of VEGF immunostaining. 


\section{Discussion}

The results of this study show for the first time that VEGF is expressed on the mRNA and protein level by chondrocytes of normal and OA human articular knee cartilage. The percentage of VEGF immunopositive chondrocytes significantly increased in severe OA cartilage. However, RT-PCR analysis disclosed a great variability in VEGF mRNA levels between cartilage samples from various donors without a clear correlation with an increased level of expression in severe OA cartilage. The discrepancy between increased immunostaining for VEGF and unaltered VEGF mRNA levels in normal and severe OA cartilage may be explained by the fact that the primers used for PCR detected all four VEGF isoforms, whereas the antibody used for immunostaining predominantly recognised the 165 amino acid long isoform of VEGF. ${ }^{14}$ Therefore, the differences of protein and RNA levels in normal and $\mathrm{OA}$ articular cartilage might reflect differential regulation of the various four isoforms of VEGF during the progression of OA.

VEGF has been found to induce capillary growth in a number of physiological and pathological conditions, including angiogenesis in growth plate cartilage and tumours. Furthermore, it has been suggested that VEGF contributes to apoptosis of hypertrophic growth plate chondrocytes by inducing the liberation of pro-apoptotic factor from blood vessels. Interestingly, in late stages of OA, blood vessel invasion from the subchondral bone into the articular cartilage has been found. ${ }^{15} \mathrm{Re}$ cently, we have shown that expression of thrombospondin-1 (TSP-1), a potent antiangiogenic factor, is decreased in OA cartilage. ${ }^{11}$ Thus it can be speculated that disturbed maintenance of pro- and anti-angiogenic factors may lead to the observed invasion of blood vessels. As mentioned above, these ingrowing blood vessels may liberate pro-apoptotic signals into articular cartilage, leading to the described apoptotic events in OA chondrocytes.

Thus this study suggests that an altered expression of VEGFs, in combination with a possible down regulation of anti-angiogenic factors in OA cartilage, may stimulate the invasion of blood vessels from subchondral bone into articular cartilage, contributing to the progression of the disease. Future work needs to establish the exact role of VEGF in normal articular cartilage, the distinct regulation of its isoforms in OA cartilage and the effect of these isoforms on invasion of blood vessels from subchondral bone, and subsequent apoptosis of chondrocytes during the progression of OA.

1 Fassbender HG. Die Arthrose-nicht nur ein degenerativer Prozeß. In: Gelenkknorpel und Arthrose. Stuttgart, Wien: Hans Huber Bern, 1982

2 Malemud CJ. Fundamental pathways in osteoarthritis: an overview. Front Biosci 1999;4:659-61.

3 Lohmander LS. Articular cartilage and osteoarthrosis. The role of molecular markers to monitor breakdown, repair and disease. J Anat 1994;184:477-92.

4 Gerber HP, Vu TH, Ryan AM, Kowalski J, Werb Z, Ferrara N. VEGF couples hypertrophic cartilage remodeling, ossification and angiogenesis during enchondral bone formation. Nat Med 1999;5:623-8.

5 Houck KA, Ferrara N, Winer J, Cachianes G, Li B, Leung D. The vascular endothelial growth factor familyidentification of a fourth molecular species and characterization of alternative splicing of RNA. Mol Endocrinol 1991;5:1806-14.

6 Ortega N, Hutchings H, Plouet J. Signal relays in the VEGF-system. Front Biosci 1999; 4:141-52.

7 Shweiki D, Itin A, Soffer D, Keseht E. Vascular endothelial growth factor induced by hypoxia may mediate hypoxiainitiated angiogenesis. Nature 1992;367:576-9.

8 Gan LM, Mioic M, Doroudi R, Selin-Sjögren L, Jern S. Factor in intact human conduit vessels exposed to laminar fluid shear stress and pressure. Biochem Biophys Res Commun 2000;272:490-6.

9 Marcus RE. The effect of low oxygen concentration in growth, glycolysis and sulphate incorporation by articular chondrocytes in monolayer culture. Arthritis Rheum 1973; 16:646-56.

10 Schneider U, Miltner O, Graf J, Niethard FU. Intraarticular oxygen partial pressure measurements under working conditions. Orthop Trans 1994;4:58.

11 Pfander D, Cramer T, Deuerling D, Weseloh G, Swoboda B. Expression of thrombospondin-1 and its receptor CD36 in human osteoarthritic cartilage. Ann Rheum Dis 2000;59: $448-54$.

12 Mankin H, Dorfman H, Lippiello L, Zarins A. Biochemical and metabolic abnormalities in articular cartilage from osteoarthritic human hips. II. Correlation of morphology
with biochemical and metabolic data. J Bone Joint Surg Am 1971;53:523-37.

13 Iizuka M, Yamauchi M, Ando K, Hori N, Furusawa Y, Itsukaichi $\mathrm{H}$, et al. Quantitative RT-PCR assay detecting the transcriptional induction of vascular endothelial growth factor under hypoxia. Biochem Biophys Res Commun 1994;205:1474-80.

14 Roberts GW, Delaat J, Nagane M, Huang S, Cavenee WK, Palade GE. Host microvasculature influence on tumor vascular morphology and endothelial gene expression. Am J Pathol 1998;153:1239-48.

15 Schiller A. Pathology of osteoarthritis. In: Kuettner KE, Goldberg VM, eds. Osteoarthritic disorders. Rosemont, IL: American Association of Orthopaedic Surgeons, 1995. 